\title{
The roles of contour and luminance distribution in determining perceived centers within shapes
}

\author{
DENNIS R. PROFFITT, M. ANNE THOMAS, and RALPH G. O'BRIEN \\ University of Virginia, Charlottesville, Virginia
}

\begin{abstract}
Two sources of visual information that likely could be employed by the perceptual system in locating the centers of bounded shapes-boundary configuration and luminance distributionhave been perfectly confounded in every study thus far aimed at investigating perceived centers. Observers, using either a revolving or stationary $x$-y plotter, made judgments on the location of centers within either revolving or stationary shapes of both uniform and varied luminance distributions. Results indicate that the location of perceived centers depended predominantly upon boundary contour and was affected by the distribution of luminance within these edges to a much lesser degree.
\end{abstract}

The visual system does not equally apprehend all points within bounded shapes, but, rather, certain locations are of special importance for perception. Two shape parameters found to be especially salient are edges and centers. Our studies assess the relative influences of two sources of visual information specifying centroid locations; however, in so doing, our findings reflect also on the perceptual importance of contours.

Hebb's (1949) proposal that edges are of special perceptual importance engendered and helped to provide theoretical significance to a considerable body of research. Studies of infants' eye movements have found the gaze of newborns to be attracted to contours (Haith, 1980; Kessen, Salapatek, \& Haith, 1972). With respect to large figures (over $10 \mathrm{deg}$ of visual angle), adults also tend to fixate edges and especially corners (Kaufman \& Richards, 1969; Zusne \& Michels, 1964). Evidence that suggests that the visual nervous system is structured to enhance and appreciate edge and corner information abounds in the neurophysiological literature (Békésy, 1967; Hubel \& Wiesel, 1962, 1968; Lettvin, Maturana, McCulloch, \& Pitts, 1959; Ratliff, 1965).

The perceptual salience of configural centroids has been found in investigations of eye fixations and motion perception. With respect to the former, Bruell and Albee (1955) and Pitts and McCulloch (1947) proposed that eye movements should be spontaneously drawn to the centroid of luminance in the visual field. This point coincides with the centroid of a bounded shape whenever the distribution of luminance is uniform within and around the figure.

This research was supported by NIH Grant MH 35530. We thank Kenneth Anderson and Robert Stoney for their assistance in running the experiments. Requests for reprints should be sent to Dennis R. Proffitt, Department of Psychology, Gilmer Hall, University of Virginia, Charlottesville, Virginia 22901.
Kaufman and Richards (1969); Richards \& Kaufman, 1969) found that spontaneous fixation tendencies were to the centroid of patterns when those figures subtended less than $5 \mathrm{deg}$ of visual angle. This finding has been supported by Coren (1981) and by Virsu (1971). With respect to motion perception, configural influences have been found to be of primary importance in determining motion percepts (Johansson, 1950), and the perceptual salience of centroids in revolving events has been repeatedly demonstrated (Börjesson \& von Hofsten, 1975; Proffitt \& Cutting, $1980 \mathrm{a})$. The focus of the present paper is on the sources of information that could be used by the perceptual system in deriving centroid locations.

\section{Perceiving the Centroid \\ of Revolving Configurations}

The effect of configuration on perceived motions was first demonstrated by Rubin (1927), who examined a situation in which various configurations of point-lights were observed to undergo complex rotational motions. The more familiar rotary motion demonstrated is that first studied by Duncker (1929/ 1938). He found that when a single point-light was mounted on the rim of an unseen rolling wheel, the motion observed was a cycloid, but that when another light was also present on the wheel, the two points were seen to move together as a perceptual group and very different motions were seen. Since Duncker's work, numerous studies have further examined this wheel-generated motion phenomenon (Börjesson \& von Hofsten, 1975; Cutting \& Proffitt, 1982; Johansson, 1973; Proffitt \& Cutting, 1979, 1980a, 1980b; Proffitt, Cutting, \& Stier, 1979; Wallach, 1965/1976). The findings of these studies can probably best be summarized graphically.

Figure la depicts the absolute motion paths of three points mounted on an unseen rolling wheel. These motions typically are not seen when viewing 
A
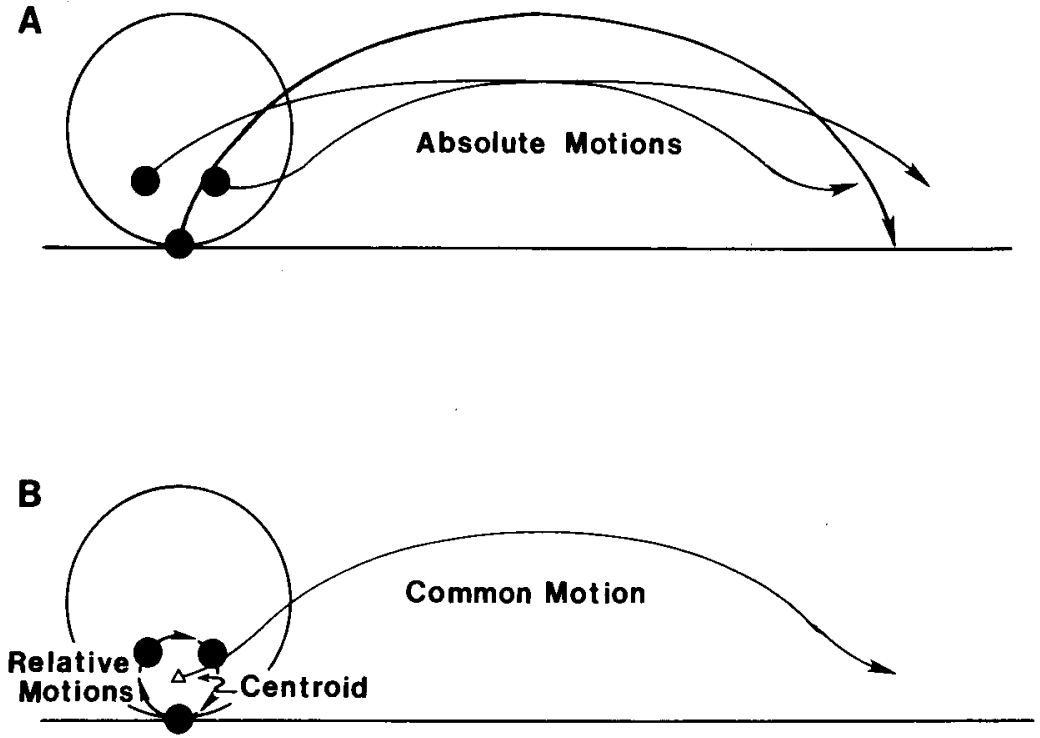

Figure 1. The top panel depicts the absolute motions of three points on an unseen rolling wheel, and the bottom panel shows the relative and common motion components that are typically percelved in this event.

this event; rather, the motions depicted in Figure 1B are more commonly observed. The perceptual system analyzes the absolute motions of the three points into two motion components. First, the relative motions of the points are seen as the rotations of each point around the centroid of their configuration. Second, the common motion of this perceptual group is seen as the motion of the centroid. These two motion components, when combined vectorially, are equivalent to the absolute motions of the individual points. The perceptual importance of the centroid is that it serves both as the center of rotation for relative motions and manifests the common motion for the configural whole. A similar analysis of absolute motions into relative and common motion components occurs when the absolute motions are rotational rather than cycloidal (Proffitt \& Cutting, 1979; Experiment 4). Figure 2A shows three points revolving on an unseen spinning disk. Figure 2B depicts a perceived analysis of the absolute circular motions into relative and common motion components. Again, the relative motions consist of rotations about the configural centroid, and the common motion consists of the centroid's motion path. In this case of a configuration on a spinning disk, the common motion of the centroid is a rotation, except for the null case that occurs when the centroid and the disk's center coincide.

\section{Two Sources of Visual Information}

\section{Specifying Centrold Locations}

Having determined that centroids are especially salient in perception, a new question surfaces: Is this perceived center the centroid of the configuration's boundary or of its luminance distribution? The former, which we call the "centroid of configuration," is determined solely from boundary information, whereas the latter, which we call the "centroid
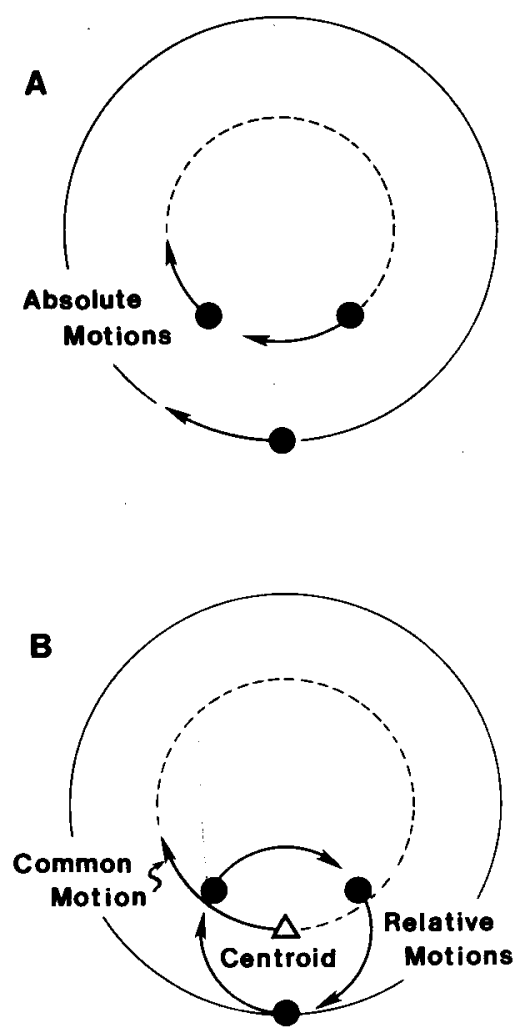

Figure 2. The top panel depicts the absolute motions of three points on an unseen revolving disk, and the bottom panel shows the relative and common motion components that are typically percelved in this event. 
of luminance," is derived in a manner that treats equally all points within and on the boundaries. These two alternatives are the most likely sources of information upon which centroid determination could depend, and they have been perfectly confounded in every study related to the role of perceived centers.

Consider the generalized shapes depicted in Figure 3. All three shapes have identical boundaries that are equivalently defined by two pairs of continuous functions. $V_{1}(x)$ and $V_{2}(x)$ define, respectively, the upper and lower boundaries between the extreme values of $a$ and $b . W_{1}(y)$ and $W_{2}(y)$ define, respectively, the right and left boundaries between the extreme values of $c$ and $d$. We define the intensity of light reflected from every point within these shapes by $f(x, y)$. For Shape $A, f(x, y)$ is a constant, whereas Shape B's and Shape C's reflectance varies across each shape.

The centroid, $\mu_{x}, \mu_{y}$, of luminance for each shape is

$$
\begin{aligned}
& \mu_{x}=\int_{a}^{b} x g(x) d x / \int_{a}^{b} g(x) d x, \\
& \mu_{y}=\int_{c}^{d} y h(y) d y / \int_{c}^{d} h(y) d y,
\end{aligned}
$$

where

$$
g(x)=\int_{V_{2}(x)}^{V_{1}(x)} f(x, y) d y, \text { and } h(y)=\int_{W_{2}(y)}^{W_{1}(y)} f(x, y) d x .
$$

Since $f(x, y)$ is a constant for Shape $A$, its value can- cels out of the centroid determination equation, $f(x, y)=1$, and

$$
g(x)=\int_{V_{2}(x)}^{V_{1}(x)} f(x, y) d y=V_{1}(x)-V_{2}(x),
$$

and

$$
h(y)=\int_{W_{2}(y)}^{W_{1}(x)} f(x, y) d x=W_{1}(y)-W_{2}(y)
$$

In words, the centroid of luminance for Shape $A$ is determined solely by boundary information and thus coincides with the centroid of configuration. More generally, whenever luminance is distributed equally within a shape, the centroids of configuration and luminance are identical. For Shapes B and C, luminance is not evenly distributed, $f(x, y) \neq 1$, and the two centroids do not coincide.

All studies showing that eye fixations are drawn to the centroid of small shapes employed stimulus materials in which the centroid of configuration and of luminance were confounded. Line drawings were used with luminance held constant within and around the contours. In the rotary motion perception studies, we find the same confounding. Proffitt and Cutting (1980b) used bounded shapes of uniform luminance in their study, and all other experiments on the role of centroids in rotational motion perception employed configurations of point-lights. Point-light arrays are typically said to define configurations with the points as the vertices. The centroids of configuration and of luminance coincide for all such arrangements.

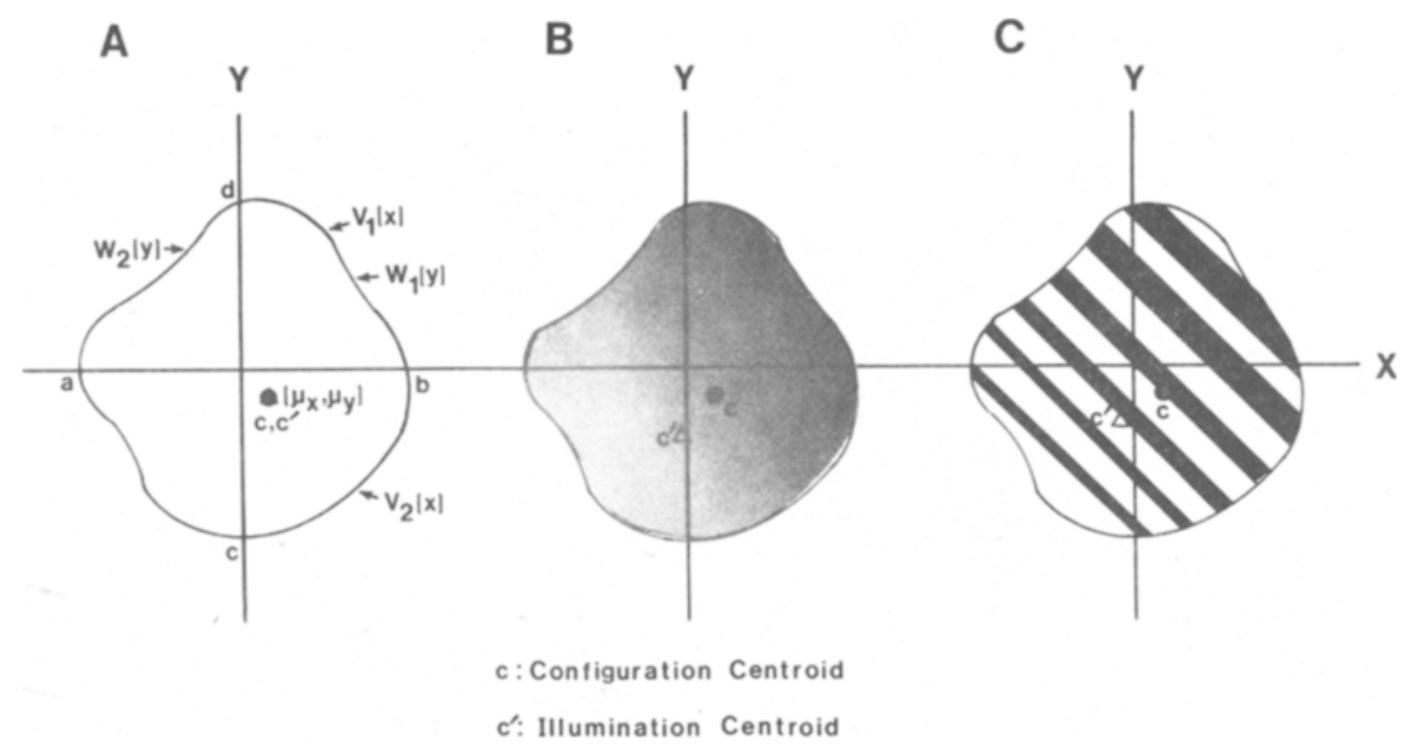

Figure 3. Depicted in panel $A$ is a shape with a uniform distribution of illumination. The shapes shown in panels $B$ and $C$ have varied luminance distributions. Only in panel $A$ do the centroids of configuration and luminance. coincide. 
Although the tenor of both the eye fixation and rotary motion perception studies is that centroids are configurally defined, the luminance alternative is favored by the two process models addressing the issue. In discussing spontaneous fixation tendencies, both Bruell and Albee (1955) and Pitts and McCulloch (1947) proposed neurophysiological models using the luminance distribution in the visual field to direct the eyes toward the centroid.

The following research was designed to assess the relative perceptual importance of boundary and overall luminance information in perceptually determining the centroids of bounded shapes. Observers were required to locate the centroid of each shape in a set of matched pairs. One member of the pair had a uniform luminance distribution, and the other was identical except for having varied luminance across its area.

\section{METHOD}

\section{The Revolving X-Y Plotter}

Described in detail by Proffitt and Simmons (1980), the apparatus consisted of two systems. The first was the revolving $x-y$ plotter, which allowed an observer to affect the location of a stimulus mounted on a revolving disk. The second system consisted of video components for the presentation of the event to the observer. A typical viewing situation is depicted in Figure 4. The observer viewed a shape revolving on the screen of a video monitor
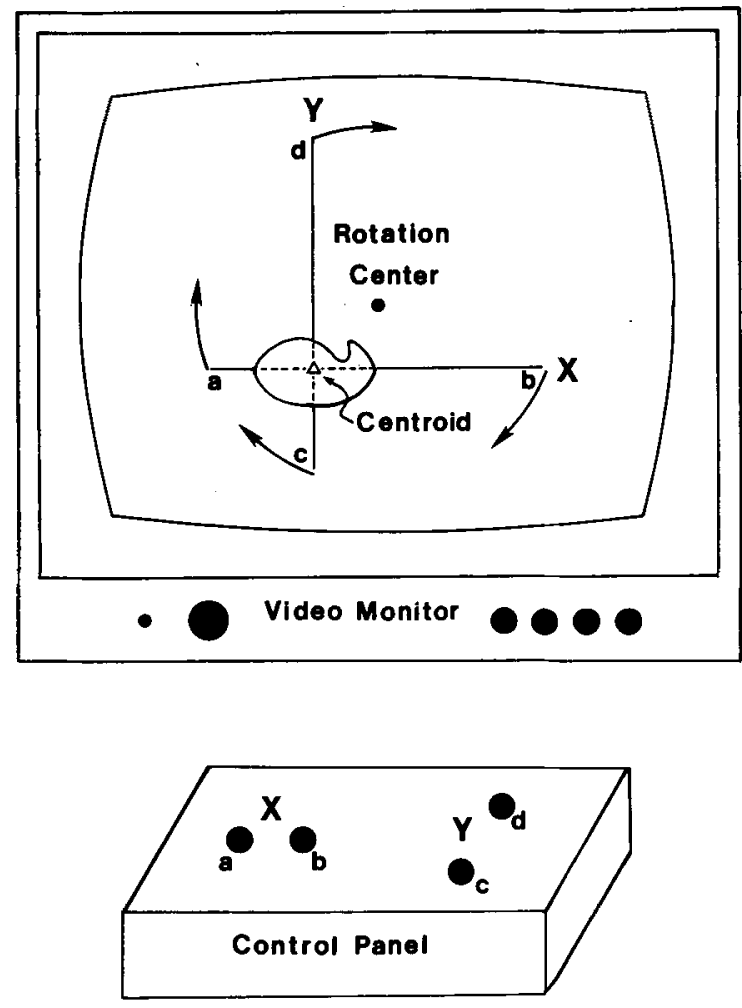

Figure 4. The viewing situation is depicted. In the experiment, only the shape is visible on the otherwise blackened video monitor screen, and the control panel is unlabeled. (700-line, 31-cm CRT). The shape was attached to an unseen $x-y$ coordinate system that rotated on an unseen disk. The observer had use of a control panel with two pairs of buttons. One pair, when pressed appropriately, caused movement of the shape in either direction with respect to the rotating $x$-axis, and the other pair worked in a like manner with respect to the rotating $y$-axis. Since these $x-y$ axes were revolving, directionality of movement had meaning relative to this rotating system, but not to the updown and left-right directions usually associated with coordinate axes. The buttons on the control panel allowed the observer to move the shape to any location on the unseen revolving disk as it rotated.

Typically, an observer was instructed to change the motion of the stimulus shape so that it appeared to spin but not to move as a whole around the screen. As previously discussed research has demonstrated, a shape revolving about any point other than its perceptual center will appear to have two motion components: a spinning motion around the perceptual center and the rotation of this abstract center point. Thus, the instructions required the observer to locate the perceived center of the shape at the origin or center of the motion-generating disk, thereby nullifying the perceived common motion component. The location within the shape that coincided with the disk's center was recorded and taken to be indicative of the perceived center of the stimulus.

\section{Stimuli}

Ten different stimuli were shown to each of the four groups of observers. These stimuli consisted of five different shapes, each seen with a uniform distribution of luminance and a luminance distribution that varied across the shape. In order to produce a systematic range of variability in observer responses, the shapes were selected so as to provide varying amounts of symmetry information specifying the configural centroid. The shapes and their order of rotational symmetry were, respectively: circle, infinite; square, four; equilateral triangle, three; oval, two; amorphous drip (depicted in Figure 4), one. As they appeared on the video monitor, the diameter of the circle, the sides of the square and the triangle, and the major axis of both the ellipse and the drip were $100 \mathrm{~mm}$. The minor axis of the ellipse was $50 \mathrm{~mm}$, and the extreme $y$-values of the drip were $\pm 25 \mathrm{~mm}$, although not orthogonal to the $x$-axis. Coordinate locations reported within these shapes are relative to the placement of the centroids at the origin and the following orientations: square, vertices on the axes; triangle, one vertex on the positive $y$-axis; ellipse and drip, $x$ - and major axis coinciding. Depending somewhat on the posture of the individual observer, the maximum visual angle subtended by each stimulus was about 5 deg.

We varied the luminance on each shape in two ways. Groups 1 , 3 , and 4 saw shapes with a luminance that was unidirectionally graded over the shape. Figure 3B depicts such a luminance distribution. This distribution was produced by covering each shape with a graduated shading film [Zipatone 10\%-90\% graduated screen tint (horizontal), 25.5 lines $/ \mathrm{cm}$ ]. The shading films were oriented on each shape so as to maximize the displacement of the luminance centroid in a negative $x$-direction without having the axes of graduation coinciding with, or falling too close to, the symmetry axes. The centroid of luminance was calculated for each shape; however, when presented on the video monitor, we could not be sure that our contrast adjustment preserved this centroid location. In order to assure that the displacement of the two centroids was equal to or somewhat greater than the calculated distances, the video monitor's contrast was increased slightly. Thus, the following coordinate locations, $(x, y)$ in millimeters, are conservative estimates of the displacement of the centroid of luminance from the configural centroid: circle, $(-3.6,0)$; square $(-4.7$, $0)$; triangle, $(-2.3, .2)$; oval $(-1.4,1.0)$; drip, $(-4.4, .5)$. Group 2 saw shapes that were made to vary in luminance by placing $5-\mathrm{mm}$ white strips on each otherwise blackened shape. Figure $3 \mathrm{C}$ depicts such a stimulus. The spacing between these strips varied uniformly from $<1 \mathrm{~mm}$ to $5 \mathrm{~mm}$. The strips were oriented in the same manner as were the shading films. The coordinate locations, $(x, y)$ 
in millimeters, for the luminance centroids were: circle, $(-5.6,0)$; square, $(-4.9, .3)$; triangle, $(-4.1, .7)$; oval, $(-8.4, .9)$; drip, $(-4.3,1.2)$.

\section{Standardizing Observations}

It is necessary to standardize observations in a manner that takes into account the dimensions of the shapes in which they are located. Consider, for example, a circle and an oval. For a circle, the range of possible point displacements from the centroid is equal in all directions; however, for an oval, observations can vary more relative to its major axis than to its minor. This implies that a standardization procedure must be employed to adjust for directional constraints on response variability. Essentially, what is required is a method that transforms all shapes into circles of equivalent areas.

We employed the method described by Proffitt and O'Brien (1982) to standardize the distances between locations in each shape and across the set of shapes. This method took advantage of descriptors common to statistical bivariate continuous density functions and to the moment descriptors previously used in visual pattern recognition and discrimination research (cf. Alt, 1962; Hu, 1962; Zusne, 1970). Using this method, each shape has the following variances and product moment correlation, $\left(\sigma_{x}^{2}, o_{x}^{2}, e\right)$ : circle, $(6.25,6.25,0)$; square, $(6.25,6.25,0)$; triangle, $(4.16,4.16,0)$; oval, $(6.25,1.56,0)$; drip, $(6.92,1.59, .06)$. Observations were standardized using the common Z-transform

$$
Z_{x}=\left(x-\mu_{x}\right) / \sigma_{x}, Z_{y}=\left(y-\mu_{y}\right) / \sigma_{y}
$$

The standardized coordinate locations $\left(Z_{x}, Z_{y}\right)$, for the luminance centroids in the shapes with graded and striped luminance distributions were, respectively: circle, $(-1.4,0 ;-2.2,0)$; square, $(-1.9$, $0 ;-2.0,0)$; triangle, $(-1.1, .1 ;-2.0, .3)$; oval, $(-.6, .8 ;-3.4, .4)$; drip, $(-1.7, .4 ;-1.6,9) .{ }^{1}$ The standardized distances, $d_{3}$, between any two points, $p_{1}$ and $p_{2}$, was computed by the following transform:

$$
\begin{aligned}
d_{s}\left(p_{1}, p_{2}\right)= & \left\{\left[\left(Z_{x_{1}}-Z_{x_{2}}\right)^{2}+\left(Z_{y_{1}}-Z_{y_{2}}\right)^{2}\right.\right. \\
& \left.\left.-2 Q\left(Z_{x_{1}}-Z_{x_{2}}\right)\left(Z_{y_{1}}-Z_{y_{2}}\right)\right] /\left(1-Q^{2}\right)\right\}^{1 / 2} .
\end{aligned}
$$

\section{Design and Procedure}

Forty University of Virginia undergraduates participated in the experiment for introductory psychology credit. Seven additional observers began the experiment; however, six failed to achieve criterion during the training phase and one chose not to complete the study. The observers were equally divided by sex among the four groups. Each observer was tested individually.

The procedure for each of the four groups was quite similar. All observers made centering responses to 10 different stimuli: five shapes, each with a uniform and a varied luminance distribution. The groups differed in either the manner in which the varied luminance was produced (graded or striped), whether or not the shapes were revolving or static, and whether or not a dot, coinciding with the center of rotation on the revolving $x-y$ plotter, was present on the video screen. The general procedure is presented under Group 1 , and modifications are discussed under the headings for the additional groups.

Group 1: Revolving shapes of uniform and graded luminance. Each observer was seen in two sessions lasting about $1 \mathrm{~h}$ each. At the beginning of the session, the observer was given training, or retraining, in the use of the revolving $x-y$ plotter. An octagon of uniform luminance was used during the training phase, and it was seen revolving around the screen with its centroid at some distance from the center of the revolving disk. After demonstrating the use of the apparatus, the experimenter instructed the observer: "Change the motion of the shape so that it appears to spin without moving as a whole around the screen."' Although becoming skilled in controlling the apparatus proved to be quite difficult for some observers, they were able to follow the instructions easily once the equipment had been mastered. We set a performance cri- terion for the training phase at three consecutive adjustments of the $x-y$ plotter deviating no more than $1 \mathrm{~mm}$ in either the $x$ - or $y$-direction from the centroid of the octagon (major axis equaling $100 \mathrm{~mm}$ ). After having reached criterion, the observer was told that he or she would be seeing 10 more events and that again the task was to make each shape spin without moving about as a whole. As with the training instructions, no mention was made of centering the shape, and the words "center" and "centroid" were never used. The instructions focused only on the nullification of common motion. Each of the five shapes was then seen in each luminance condition. The order of their presentation was randomized, although under the condition that the same shape was never seen on consecutive trials. Following completion of this task, the observer was asked to come back on another day, and at that time the task was repeated with a different ordering of the 10 events. The observer's responses were averaged across these two sessions.

Group 2: Revolving shapes of uniform and striped luminance. The procedure for Group 2 was identical to that for Group 1. These groups differed only in that Group 2 observed the striped shapes rather than the graded ones.

Group 3: Static shapes of uniform and graded luminance centered under a dot. The procedure for Group 3 was similar to that for Group 1, except that the shapes were not revolving. Each shape was initially seen on the video screen at some distance from a small dot placed slightly off center on the monitor. The observer was instructed: "Move the shape so that its center falls directly under the dot." The two pairs of control buttons caused the shape to move in orthogonal directions. One pair of buttons produced movement in a 125- or 305-deg direction, whereas, the other produced movement in a 35- or 115-deg direction. The shape was in motion only when the observer was making adjustments of its position on the screen.

Group 4: Revolving shapes of uniform and graded luminance centered under a dot. In order to compare performance in the above static condition with that of the observers viewing revolving displays, Group 4 replicated the procedure on Group 1, except that a dot was present on the screen. The instructions were not changed, and little mention was made of the dot. The relationship between the dot and the center of rotation for the display was not pointed out to the observers; however, this relationship became obvious during the training phase.

\section{RESULTS AND DISCUSSION}

Our experimental procedure yielded two performance measures: mean location of perceived centers and variability among individual placements. The first measure is necessarily directional, and Figures 5 , 6,7 , and 8 present, for each group, the mean centroid relative placements, in standardized Z-scores, on the $x$-axes. Since the luminance centroids were displayed almost exclusively in the negative $\mathrm{x}$-direction, the $y$-axis placements are not depicted. As a measure of variability for the shape and group in question, we used the mean standardized distance, $d_{s}$, between each placement and the observed sample centroid (group mean). These values are presented, for each group, in Figure 9. Our repeated measures design was analyzed using a multivariate analysis of variance strategy, employing the Pillai-Bartlett criterion with appropriate conversion to an approximate $\mathrm{F}$ statistic. We used a Bonferroni strategy to control familywise Type I error rates. For example, if a question required four separate tests, we adopted a $.05 / 4=.0125$ pretest significance level. 
Group 1

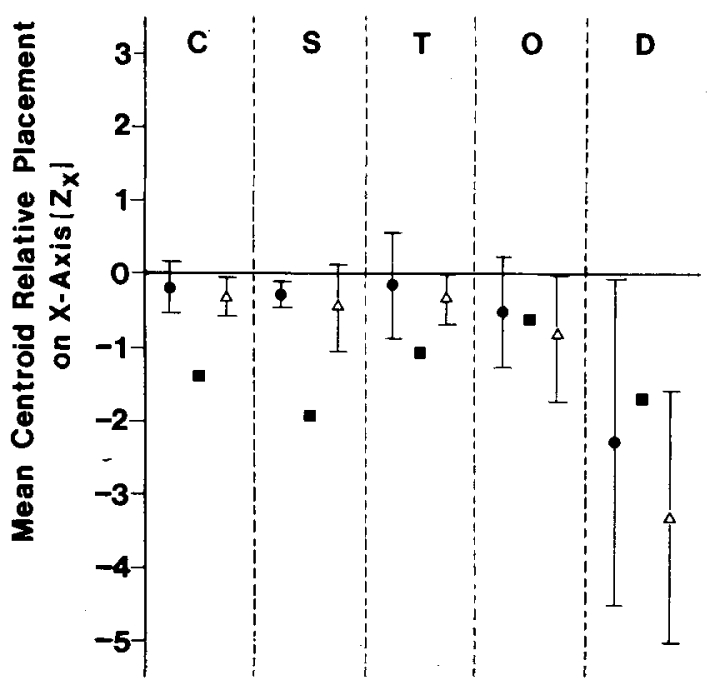

- Uniform Luminance

$\triangle$ Varied Luminance

- Centroid of Varied Luminance

Figure 5. Group 1 mean x-axis locations, in Z-scores, of the perceived centers relative to the configural centroids. Shapes are dealgnated: $C$, circle; S, square; T, triangle; O, oval; D, drip. Plus and minus $95 \%$ confidence intervals are given by vertical hatches.

It can be seen by examining Figures 5 through 8 that, except for the drip, means relative to the $x$ axis for all placements within uniformly illuminated shapes fell quite close to their centroids. Across shapes and groups, all placements relative to the $y$ axis were essentially on the configural centroid as well. Noting the exception, this finding corroborated the results of Proffitt and Cutting (1980b). The perceived center of the drip was displaced negatively on its $x$-axis: mean centroid relative placement on the $x$ axis for the drip differed significantly from the other four shapes over all groups $[F(1,36)=28.59, p<$ $.001]$ and within Groups 1 and $2[F(1,36)=17.80$, $\mathrm{p}<.001 ; \mathrm{F}(1,36)=9.69, \mathrm{p}=.004 ; \mathrm{F}(1,36)=1.73$, n.s.; $F(1,36)=4.17$, n.s.; for Groups $1-4$, respectively]. As in the other groups, mean location of the perceived center in Groups 3 and 4 was negatively displaced on the $\mathrm{x}$-axis; however, the magnitude of its displacement was less in these situations.

We expected variability to increase inversely with order of symmetry across the five shapes as evidenced in Figure 9. The analysis of the linear trend was significant $[F(1,36)=159.28, p<.001]$. The four groups did not differ significantly with respect to this trend.

The luminance manipulation caused perceived centers to be slightly displaced away from configural centroids and toward luminance centroids in the revolving conditions (Groups 1, 2, and 4) but not in the static situation (Group 3). Since the displacement of

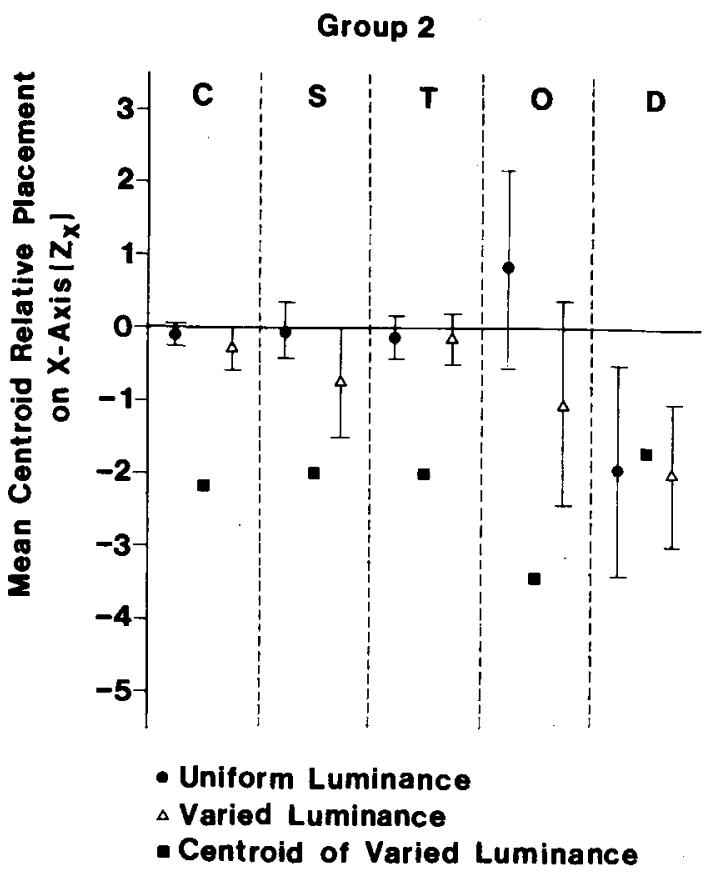

Figure 6. Group 2 mean $x$-axis locations, in $Z$-scores, of the perceived centers relative to the configural centroids. Shapes are designated: C, circle; S, square; T, triangle; O, oval; D, drp. Plus and minus $95 \%$ confidence intervals are given by vertical hatches.

the luminance centroid from the configural centroid was primarily in the $x$-direction, we anticipated the finding that, averaged across shapes and groups, the luminance conditions significantly differed only on the $x$-axis $[F(1,36)=10.40, p=.003]$. Although the perceived centers for the varied luminance shapes were displaced toward their luminance centroids, mean placements were still closer to the configural centroids. An examination of Figures 5 through 8 reveals that, excluding the drip (for which meaningful comparisons cannot be made due to the negative $x$ displacement of its perceived center in the uniform luminance condition), the perceived center was located on the $x$-axis reliably closer to the configural centroid 10 times and reliably closer to the luminance centroid only once. As will be discussed later, the static condition in Group 3 showed no effects of the luminance manipulation.

As Figure 9 shows, the luminance manipulation increased response variability. This was found to be the case over all of the groups $[F(1,36)=7.79, p=.008]$, and the individual groups did not differ from each other in this respect.

We created a range in response variability across shapes in order to determine whether or not the luminance manipulation would interact with this variability. That is, we thought that as the configural symmetry information decreased across the set of shapes, the displacement toward the luminance centroid might increase. A significant interaction on 
Group 3

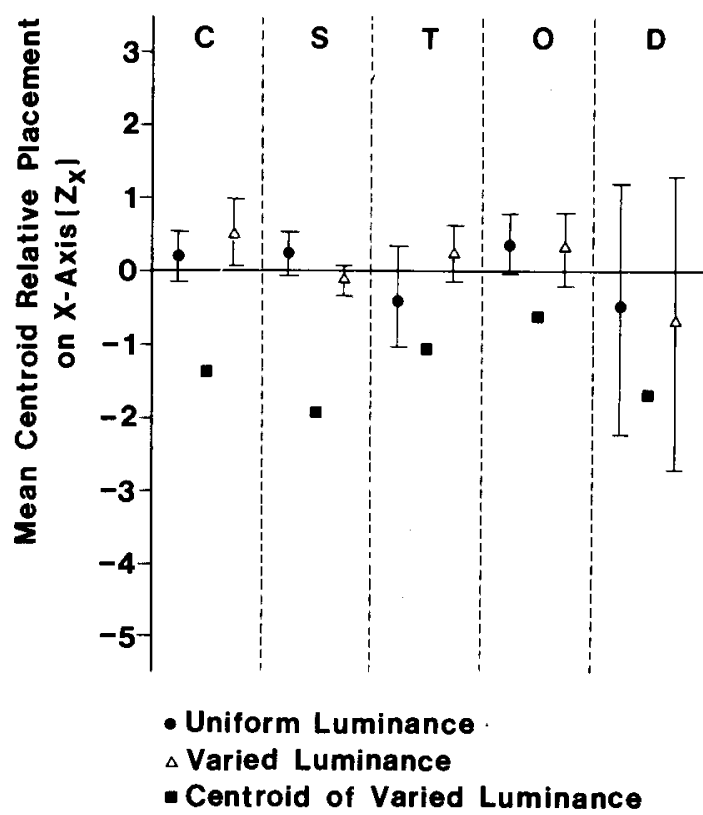

Figure 7. Group 3 mean $x$-axis locations, in Z-scores, of the percelved centers relative to the configural centroids. Shapes are designated: $C$, circle; S, square; T, triangle; O, oval; D, drip. Plus and minus $95 \%$ confidence intervals are given by vertical hatches.

mean placements with respect to the $\mathrm{x}$-axis was found between shape and luminance conditions $[F(4,33)=$ $4.25, \mathrm{p}=.007]$; however, this interaction was not of the expected pattern. Except when the circle was compared with the other shapes, we did not find that displacement toward the luminance centroid increased with decreasing symmetry. The circle significantly differed from the other three shapes having nontrivial symmetry in that the luminance manipulation for the circle was ineffective in inducing a shift toward its luminance centroid $[F(1,36)=11.03, p=$ $.002]$. The other shapes did not differ in mean luminance effects. The special status of the circle can be understood by recognizing that when rotating around its centroid, a circle of uniform luminance appears not to be moving at all. The circumference of the varied luminance circle also remains stationary under centroid relative rotation, and even slight displacements from this point yield dramatic perceptions of wobbling motion.

The final set of results to be discussed is the differential effects found for the four groups. In essence, there were no differences between the three groups that viewed the revolving displays; however, the group that viewed the static display differed from its control. Comparing the four groups on mean centroid relative displacements over all shapes, there were no significant differences, and no differences were found relative to response variability. As previously mentioned, the luminance manipulation was found to interact with groups. The three revolving

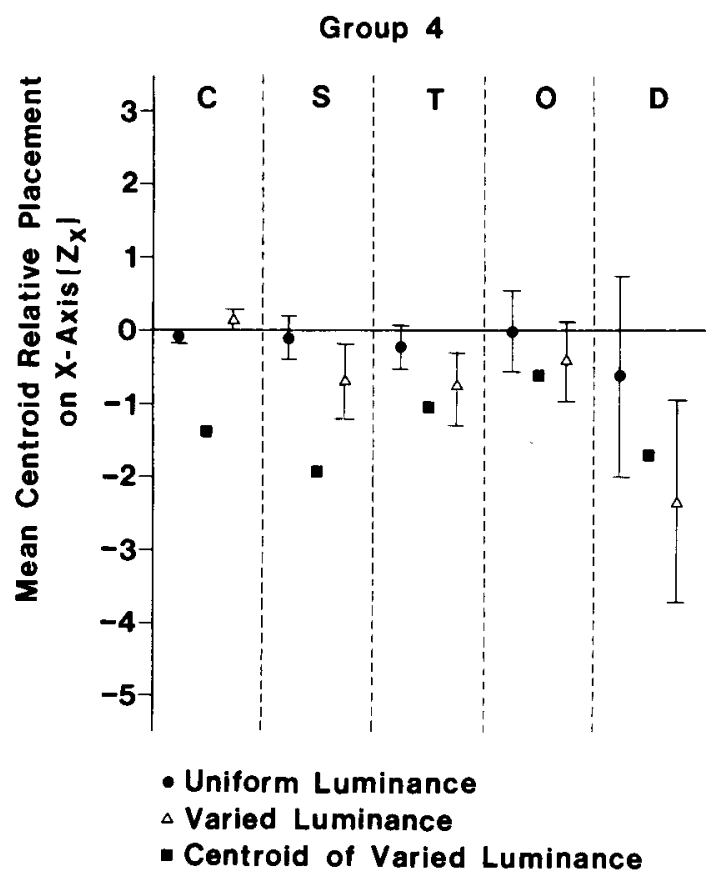

Figure 8. Group 4 mean x-axis locations, in Z-scores, of the percelved centers relative to the configural centroids. Shapes are designated: $C$, circle; $S$, square; T, triangle; O, oval; D, drip. Plus and minus $95 \%$ confidence intervals are given by vertical hatches.

conditions did not differ significantly in this regard; however, Group 3 was found to be unaffected by this manipulation and differed significantly in this lack of displacement along the $x$-axis from its control, Group $4[F(1,36)=5.99, p=.02]$, as well as from the other three groups combined $[F(1,36)=6.14$, $\mathrm{p}=.02]$. The luminance manipulation did not produce differential response variability among the four groups.

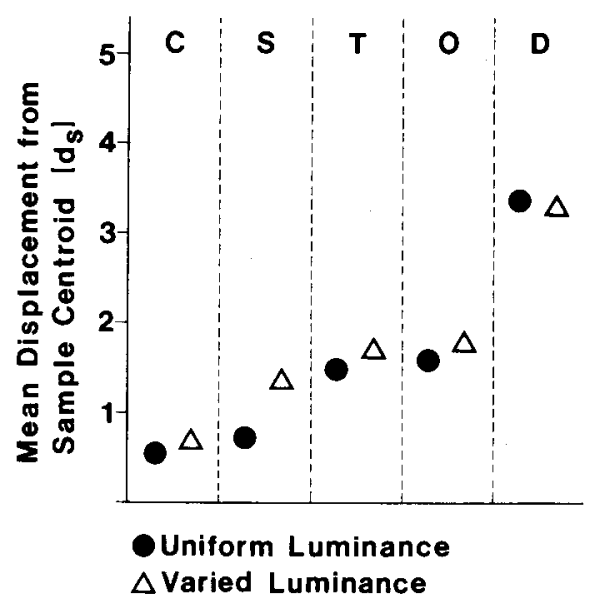

Figure 9. The mean standardized displacements, $d_{3}$, of the perceived centers from their sample centroids. Displacements are averaged across groups. Shapes are designated: $\mathrm{C}$, circle; $\mathrm{S}$, square; $T$, triangle; $O$, oval; $D$, drip. 
To recapitulate, the luminance manipulation shifted the perceived center of revolving shapes from the configural centroid toward the centroid of luminance and increased response variability in both revolving and static conditions. Although somewhat displaced, the perceived center in varied luminance shapes undergoing rotation was still closer to the configural than to the luminance centroid. That the static condition was not affected by the luminance manipulation may reflect a greater salience of configural information in this situation. The luminance manipulation did not demonstrate increasing effects as configural symmetry information decreased across the set of shapes. This implies that the two sources of information specifying centroid locations-contour configuration and luminance-were either independent in their influences or too weakly interactive for our procedures to detect. Finally, the only reliable difference found among the groups was between those who saw static rather than revolving displays. Group 3 was unaffected by the luminance manipulation.

\section{CONCLUSION}

In determining the perceptual center of shapes, the visual system relies predominantly on boundary information. Of two possible sources of information upon which centroid determination could dependboundary configuration and luminance distributionthe centroid defined by the former corresponded far more closely to the perceived center than did that specified by the latter. This was found to be the case in both static and moving displays.

Although centroid determination seems to be based upon boundary information, it is the contour of the shape that is salient, and not simply edge information. The shapes shown to Group 2 consisted of stripes falling both on and within the boundary of each form; however, it was primarily the perimeter boundary that specified the perceived centers observed in these shapes. A centroid calculated from all edge information in striped shapes corresponded to the luminance centroid, whereas the configural centroid utilized only the perimeter boundary information. (The perimeter boundary is defined as including the subjective contours between the perimeter edges of the stripes.) The location of perceived centers for shapes depended predominantly upon their boundary contour and was affected by the distribution of luminance within these edges only to a much lesser degree.

The differences found between the groups that viewed the moving and static displays reflects upon the often made claim that perceiving static forms ought to be considered a special case of dynamic perception (Cutting \& Proffitt, 1981; Gibson, 1979; Johansson, 1974). This assertion may require quali- fication, given that we did not find a greater accuracy in locating the center in the revolving, relative to the static, displays. Börjesson and von Hofsten (1975) and Cutting and Proffitt (1982) proposed that the perceptual system, in selecting the centroid as the perceived center, used a dynamic minimum principle to nullify relative rotational motions. Thus, there was configural and motion information available in dynamic perception, but only configural information was available in perceiving static shapes. Either the motion information was not used or, in perceiving revolving shapes, some configural information was lost. Since we know from studies on perceiving unbounded and nonrigid shapes (cf. Cutting \& Proffitt, 1982) that motion information can be used to determine perceived centers, it is most likely the case that some configural information is lost when the revolving shapes were viewed and that the additional motion information afforded by these displays compensated for that deficit. Whether or not this conjecture is supported by future research, the special status of static perception within dynamic perception is brought into question by the lack of a facilitating influence of rotation on centroid determination. (The slight, but reliable, luminance effect found in revolving but not static displays also reflects on this issue; however, instruction differences may have produced this result.)

That perceiving shapes is selective, with boundary edges and centroids being especially salient, has been repeatedly demonstrated. The present study showed that the special status of centers owes its location primarily to the special status of boundary contours. Our manipulation to vary the luminance distribution within shapes did little to affect the location of perceived centers from their configurally specified locations.

\section{REFERENCES}

Alt, F. L. Digital pattern recognition by moments. In O. L. Fischer, Jr., D. K. Pollack, B. Raddack, M. E. Stevens (Eds.), Optical character recognition. Washington, D.C: Spartan Books, 1962.

Béḱ́sy, G. von Sensory inhibition. Princeton, N.J: Princeton University Press, 1967.

Böruesson, E., \& von Hofsten, C. A vector model for perceived object rotation and translation in space. Psychological Research, 1975, 35, 209-230.

Brueli, J. H., \& Albee, G. W. Notes toward a motor theory of visual egocentric localization. Psychological Review, 1955, 62, $391-400$.

Conen, S. The interaction between eye movements and visual perception. In D. F. Fisher, R. A. Monty, \&. W. Senders (Eds.), Eye movements: Cognition and visual perception. Hillsdule, N.J: Erlbaum, 1981.

Cutrina, J. E., \& Profritr, D. R. Gait perception as an example of how we may percelve events. In R. D. Walk \& H. L. Pick (Eds.), Intersensory perception and sensory integration. New York: Plenum, 1981.

Cutring, J. E., \& Proprits, D. R. The minimum principle and the perception of absolute, common, and relative motions. Cognitive Psychology, 1982, 14, 211-246. 
Duncker, K. [Induced motion.] In W. D. Ellis (Ed. and trans.), A sourcebook of Gestalt psychology. London: Routledge \& Kegan Paul, 1938. (Originally published, 1929.)

Gibson, J. J. The ecological approach to visual perception. Boston: Houghton Mifflin, 1979.

Haith, M. M. Rules that babies look by. Hillsdale, N.J: Erlbaum, 1980.

HeBB, D. O. Organization of behavior. New York: Wiley, 1949.

Hu, M.-K. A mathematical model for visual perception. In E. E. Bernard \& M. R. Kare (Eds.), Biological prototypes and synthetic systems. New York: Plenum Press, 1962.

Hubel, D. H., \& Wiesel, T. N. Receptive fields, binocular interaction and functional architecture in the cat's visual cortex. Journal of Physiology, 1962, 160, 106-154.

HubeL, D. H., \& WIESEL, T. N. Receptive fields and functional architecture of monkey striate cortex. Journal of Physiology, $1968,195,215-243$.

Johansson, G. Configuration in event perception. Uppsala: Almqvist \& Wiskell, 1950.

Joh Ansson, G. Visual perception of biological motion and a model for its analysis. Perception \& Psychophysics, 1973, 14, 201-211.

Johansson, G. Projective transformations as determining visual space perception. In R. B. MacLeod \& H. L. Pick (Eds.), Perception: Essays in honor of James J. Gibson. Ithaca, N.Y: Cornell University Press, 1974.

KaUfman, L., \& Richards, W. Spontaneous fixation tendencies for visual forms. Perception \& Psychophysics, 1969, 5, 85-88.

Kessen, W., Salapatek, P., \& Haith, M. M. The visual response of the newborn to linear contour. Journal of Experimental Child Psychology, 1972, 13, 9-20.

Letrvin, J. Y., Maturana, H. R., McCulloch, W. S., \& Pitts, W. H. What the frog's eye tells the frog's brain. Proceedings of the IRE, 1959, 47, 1940-1951.

Pitrs, W., \& McCulloch, W. S. How we know universals: The perception of auditory and visual forms. Bulletin of Mathematical Biophysics, 1947, 7, 89-93.

Proffitt, D. R., \& Cutrina, J. E. Perceiving the centroid of configurations on a rolling wheel. Perception \& Psychophysics, $1979,25,389-398$.

ProffitT, D. R., \& Cutring, J. E. An invariant for wheelgenerated motions and the logic of its determination. Perception, 1980, 9, 435-449. (a)

Proffitt, D. R., \& Cutrina, J. E. Perceiving the centroid of curvilinearly bounded rolling shapes. Perception \& Psychophysics, 1980, 28, 484-487. (b)

Proffitt, D. R., Cutring, J. E., \& Stier, D. M. Perception of wheel-generated motions. Journal of Experimental Psychology: Human Perception and Performance, 1979, 5, 289-302.

Proffitt, D. R., \& O'Brien, R. G. Standardizing distances of observations within shapes. Perception \& Psychophysics, 1982, 32, 302-305.

Proffitt, D. R., \& Simmons, R. W. A revolving X-Y plotter for studying the perception of rotational motions. Behavior Research Methods \& Instrumentation, 1980, 12, 601-604.

RATLIFF, F. Mach bands: Quantitative studies on neural networks in the retina. San Francisco: Holden-Day, 1965.

Richards, W., \& KaUfman, L. "Center-of-gravity" tendencies for fixations and flow patterns. Perception \& Psychophysics, $1969,5,81-84$.

Rubin, E. Visuell wahrgenommene wirkliche Bewegungen. Zeitschrift für Psychologie, 1927, 103, 384-392.

VIrsu, V. Tendencies to eye movement, and misperception of curvature, direction, and length. Perception \& Psychophysics, 1971, 9, 65-72.

Wallach, H. Visual perception of motion. In G. Keyes (Ed.), The nature of the art of motion. New York: Braziller, 1965. (Also in H. Wallach. On perception. New York: Quadrangle, 1976).

Zusne, L. Visual perception of form. New York: Academic Press, 1970.

Zusne, L., \& Michels, K. M. Nonrepresentational shapes and eye movements. Perceptual and Motor Skills, 1964, 18, 11-20.

\section{NOTES}

1. Displacement of the luminance centroid necessarily varied across shapes because the rate of luminance change was constant. In order to make displacements equivalent, the luminance distributions would have had to vary at markedly different rates across the shapes.

2. Since the octagon was of uniform luminance, the training procedure did not create a response bias favoring either centroid location.

(Manuscript received May 24, 1982; revision accepted for publication October 26, 1982.) 